\title{
CÓMO ESCRIBIR EL INFORME FINAL DE UNA TESIS
}

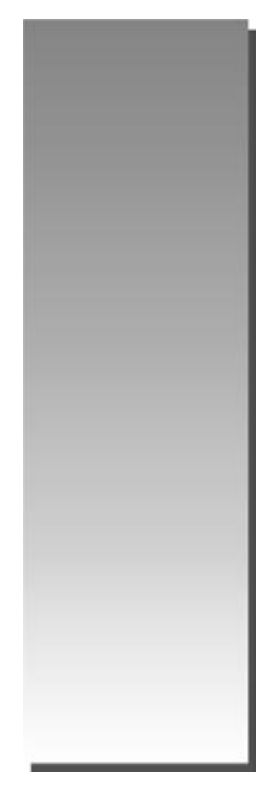

Pedro L. Tito Huamani *

E-mail: pedrotito18@hotmail.com

\section{RESUMEN}

El informe final de la tesis de investigación se vuelve un dolor de cabeza para la mayoría de egresados de maestrías y doctorados, debido a que deben presentar de manera organizada y sistematizada el tema investigado, describiendo paso a paso la tarea realizada. En el caso de las ciencias administrativas, uno de los mayores limitantes para la presentación de estos documentos es la falta de modelos o guías, por lo que el presente texto brinda algunas pautas para ayudar a los egresados de maestrías y doctorados en la elaboración de su informe final de investigación.

Palabras clave: Tesis de investigación, informe final de investigación, estructura del informe final de investigación.

\section{ABSTRACT}

The final report of the thesis research is becoming a headache for most graduates of masters and doctoral degrees, due to be presented in an organized and systematic investigated the issue, describing step by step the task done. In the case of administrative sciences, one of the major constraints for the submission of these documents is the lack of role models or guides, so that this text provides some guidelines to help graduates of master's and doctoral degrees in the development of the final investigation report.

Key words: Thesis research, Final report of investigation, structure of the final report of investigation. 


\section{INTRODUCCIÓN}

Cuando un egresado de una universidad decide utilizar la vía de la presentación de tesis para optar su grado académico, uno de los problemas que debe solucionar es la forma cómo estará estructurado su informe final. Se entiende que este es el informe de la investigación que llevó a cabo observando lo descrito en su proyecto o plan de investigación, previamente aprobado.

En ese contexto, ningún proyecto se considera completo hasta que se prepara el informe final de investigación; incluso el estudio más brillante tiene poco valor sino se difunde a la comunidad científica. El informe de investigación representa el resultado final del largo proceso de investigación. Su articulación estructural es el modo como los investigadores ordenan, clasifican y presentan los datos.

Cualquiera sea el tipo de investigación, la presentación de los resultados se hace sobre la base de normas que permiten estructurar, de una manera lógica, la forma y el contenido de la exposición teórica. El objetivo del presente artículo es brindar algunos lineamientos generales para ayudar a los egresados de áreas empresariales a comunicar los resultados de su investigación. Los antecedentes que tenemos demuestran que existe una limitante en el ámbito estudiantil sobre la obtención de modelos o guías que les permitan presentar los resultados que se han obtenido en una investigación. Como su nombre lo dice, este artículo viene estructurado de tal forma que el egresado podrá desarrollar paso a paso el informe final de su investigación.

Básicamente todo informe final de investigación, traducido en tesis de grado académico, está compuesto por: Portada, Preliminar (Dedicatoria y Agradecimiento, Índice, Resumen, Introducción), Cuerpo del Informe, Conclusiones, Recomendaciones, Anexos y Bibliografía. Sin embargo, en este informe se han agregado otros elementos que complementan la oportuna comunicación de los resultados.

\section{SUGERENCIAS INICIALES DE FORMA PARA LA PRESENTACIÓN DE LA TESIS.}

- Tipo de letra: arial 12, estilo normal, color negro, justificado y a doble espacio entre líneas. En este último, hay algunos que prefieren hacerlo a espacio y medio, o incluso a un espacio.
- Tamaño de papel: bond A4, medidas $210 \mathrm{~mm}$. x 297 mm., 75 u 80 grs., base 20 .

- Márgenes: Se sugiere respetar en lo posible los que a continuación se indican:

$\begin{array}{lll}\text { - } & \text { Superior : } & 4.0 \mathrm{~cm} . \text { (1.2 pulgadas). } \\ \text { - } & \text { Inferior : } & 2.5 \mathrm{~cm} . \text { (1 pulgada). } \\ \text { - } & \text { Izquierda : } & 3.5 \mathrm{~cm} . \text { (1.05 pulgadas). } \\ \text { - } & \text { Derecha : } & 2.5 \mathrm{~cm} . \text { (1 pulgada). }\end{array}$

- En la práctica, muchos informes tienen otras medidas, pienso más por una conveniencia del informante.

- En concordancia con el segundo punto, en cada página se deben escribir 24 reglones que deben ser enumerados en la esquina inferior derecha, con números arábigos en el orden correlativo correspondiente. El número debe aparecer al menos a $2.5 \mathrm{~cm}$ (1 pulgada) de la orilla derecha de la página, en el espacio entre el borde inferior del documento y la primera línea del texto.

- El índice, resumen y la introducción se enumeran en el orden i, ii, iii. El cuerpo del trabajo se enumera siguiendo el orden correlativo acostumbrado.

\section{LA PORTADA}

La portada es la parte inicial del informe de investigación, que está compuesta por los siguientes elementos que van centrados en la página:

- A nivel de pregrado: Universidad, Facultad y Escuela Académico Profesional. A nivel de postgrado: Universidad. Escuela de Post Grado. Unidad de Post Grado de la Facultad pertinente.

- Logo de la universidad

- Título de la tesis (investigación)

- Mención del grado académico al que conduce la tesis.

- Nombres y apellidos del autor.

- Mes y año de presentación.

\section{PRELIMINAR}

\section{Dedicatoria y Agradecimiento}

Es costumbre en nuestro medio dedicar y agradecer al ser supremo, familiares y personas que el estudiante aprecia, situaciones, hechos, etc. que coadyuvaron a la realización 
de la investigación. Se sugiere no utilizar más de dos hojas.

\section{2. Índice}

Es un listado de las partes estructurales del informe de investigación; se coloca después de la portada, dedicatoria y agradecimiento pero antes del resumen. Incluye los capítulos y subcapítulos, temas y subtemas que son y forman parte de la totalidad de la investigación. Se señalan las partes y el número de página correspondiente.

El lector, al consultar el índice, se pone en contacto con todo el contenido del escrito, lo que facilita la localización de los temas generales y específicos. La forma que se sugiere adoptar para el índice del trabajo de investigación será la llamada sistema general, en el que se utilizan números romanos para identificar los capítulos; letras mayúsculas para los temas, números arábigos para los sub temas y letras minúsculas para las subdivisiones de éstos.

\section{Resumen}

También se conoce como abstract. En él se detalla sintéticamente todo el contenido del informe de investigación, planteando las ideas centrales y el perfil del escrito. Se busca reducir a términos breves y precisos la idea central de la investigación realizada. Debe incluir la justificación, objetivo general, principales resultados y conclusiones. Su extensión varía desde una hasta dos hojas como máximo.

\section{Introducción}

Es la comunicación inicial que permite (sin necesidad de explicar) penetrar en detalles incentivadores, creando un ambiente de familiaridad y confianza entre el autor del escrito y la persona lectora. Además de ser incentivadora, la introducción presenta el tema de investigación, los propósitos esenciales y datos generales del contenido estructural del escrito; es decir, hace una breve descripción capitular.

Las páginas de la introducción se numeran independientemente del resto del texto con números romanos, los que deben colocarse en la parte inferior derecha. El texto no debe exceder de diez hojas.

Para redactar la introducción deberán cumplirse las siguientes recomendaciones:
1. Debe ser clara y concreta.

2. Redactarla al finalizar el ordenamiento y clasificación de todos los datos, es decir, cuando se ha terminado de elaborar el informe final.

3. Se ubica luego de la tabla de contenidos o índices.

4. Prepara al lector para la descripción de lo que se hizo.

5. Se menciona el tema de investigación y los objetivos. Describe el estudio, la explicación del marco conceptual, las hipótesis y la justificación.

6. Se presentan los resultados y definiciones más importantes, en forma resumida.

7. Es bueno articular en forma lógica la presentación (¿qué es el escrito?, ¿cuál es el título?, ¿a qué institución se presentará?, ¿quiénes serán los lectores?), el propósito, las partes estructurales generales y una breve metodología de exposición.

\section{CUERPO DEL INFORME}

\section{Capítulo I: El problema}

Es la descripción del problema que puede sustentarse en el desconocimiento de las causas que lo generan, los factores asociados o el grado de intensidad mostrado en el comportamiento de algunos indicadores o variables en un contexto determinado. Para los estudiantes de Administración, se entiende que el problema estará enmarcado en un contexto organizacional

Puede subdividirse en los siguientes apartados:

\subsection{Planteamiento del problema (relacionado al tema de estudio)}

En este apartado puede plantearse:

- Breve antecedente del problema. (Antecedentes del problema)

- Síntomas que la reflejan. (Situación problemática)

- Efectos inmediatos y futuros de continuar subsistiendo el problema.

- Causas probables. Factores asociados.

- Datos que verifiquen que el problema es parte de un contexto en el que se conjugan otros problemas relativos. 
- Actores y/o instituciones involucradas.

- Soluciones que se han intentado.

- Interrogantes fundamentales, preguntas a responderse en la investigación. (Enunciado del problema)

Todo lo anterior debe ser redactado en forma lógica y coherente, en el entendido que el enfoque utilizado es deductivo. Con la misma rigurosidad académica se debe actuar si la investigación responde a un enfoque inductivo, y tanto mejor si es abductivo.

\subsection{Formulación y sistematización del pro- blema}

Son aquellas interrogantes que el investigador se plantea al definir el problema a investigar. Tales preguntas son importantes para establecer los objetivos y las hipótesis de la investigación.

\subsection{Delimitación de la investigación.}

En este apartado se establecerá descriptivamente la cobertura que tuvo la investigación en lo relativo a:

- Espacio geográfico, es decir, el lugar u organización donde se realizó la investigación.

- Sujetos y/u objetos que participaron en la realización del estudio.

- Tiempo, especificando el periodo de tiempo en el que se realizó la investigación.

- Contenidos, se deben mencionar las variables que se consideraron en el estudio.

\subsection{Justificación}

Este apartado debe reflejar la importancia y relevancia que tiene la investigación que se ha realizado, exponiendo argumentos tales como:

- Evidencias que demuestren la magnitud de la problemática o necesidad de éstas para profundizar en el análisis.

- Necesidad de corregir o diseñar medidas correctivas que contribuyan a la solución de los problemas expuestos.

- Demostrar que la investigación constituye una estrategia para enfrentar la problemática mencionada.
- Mencionar los beneficios futuros que pueden obtenerse, tanto para las personas como para las instituciones y/o grupos sociales.

\subsection{Limitaciones}

Este apartado debe reflejar las restricciones que tiene la investigación para poder expandir o generalizar los resultados, así como el reconocimiento de las incidencias de otras variables que en el proceso de la investigación no se controlan. Se recomienda evitar mencionar limitantes que puedan preverse antes de realizar la investigación, tales como tiempo, costos, falta de información y otras propias de los investigadores.

\subsection{Objetivos}

- Deben expresarse como proposiciones orientadas a definir los logros que se esperan obtener a partir de los resultados que arroje la investigación.

- Los elementos que lo constituyen son el verbo, la(s) variables, la(s) relación(es) y el contexto.

- Deben reflejar lo que se espera obtener al estudiar cada variable (cuando no se establezca una relación bivariada), o lo que se espera al estudiar la relación de dos o más variables.

- Debe formularse un objetivo general y varios específicos, en donde estos últimos sean desglosados del primero.

\section{Capítulo II: Fundamentación Teórica.}

En este capítulo se exponen algunos principios, axiomas o leyes que rigen las relaciones de los fenómenos que son parte de la realidad estudiada y que de alguna manera están incidiendo o son parte de la casualidad o estructura lógica de las explicaciones teóricas de alguna disciplina científica

Este capítulo debe estructurarse dependiendo de la naturaleza de cada investigación, pero en términos generales deben considerarse aspectos tales como:

- Breve marco histórico.

- Contexto en el que están inmersas las variables del problema.

- Síntesis del marco social, cultural, legal, institucional. 
- Comportamiento de las variables en otros ambientes y contextos.

- Enfoques de autores o teorías que las respaldan.

- Relación de las variables.

Todo lo anterior debe plantearse bajo un enfoque deductivo, es decir desde lo más general hasta lo más específico, a menos que el tipo de investigación demande otro tipo de enfoque.

Así también cada aspecto debe ser comentado e interpretado, combinando las ideas propias del investigador con las ideas de otros autores. Esto último tiene el propósito de reforzar nuestros planteamientos a partir de los argumentos de ilustrados que ya investigaron o son referentes en el mundo académico.

Con respecto a las referencias bibliográficas, es indistinto usar las numeraciones clásicas que jalan a las precisiones del autor en la nota o pie de página o usar las citas cortas. En este último, por ejemplo, una referencia puede ser: (Drucker, 6) o $(3,6)$ en donde 3 corresponde al tercer libro de la bibliografía y 6 corresponde al número de la página en donde está ese párrafo textual.

Cualquiera sea la modalidad utilizada en las referencias bibliográficas, lo importante de ello va por el lado de guardar la coherencia de la modalidad desde el inicio hasta el final de informe presentado.

\section{Capítulo III. Hipótesis y Variables}

En este capítulo deben expresarse los supuestos básicos que orientan la búsqueda de la información y cómo éstos han sido operacionalizados a través de la relación esperada de las variables. De igual manera deben exponerse los indicadores que permitirán medir el comportamiento de las variables a evaluar.
Los apartados sugeridos son:

- Hipótesis Generales y Específicas.

- Diagrama de variables.

- Indicadores de las variables.

- Operacionalización de variables.

\subsection{Hipótesis Generales y Específicas}

En este apartado se sugiere formular las hipótesis de investigación, tal como estaban en el proyecto (plan) de investigación. De ser necesario, éstas deben correlacionarse con cada objetivo (solamente cuando la investigación demande efectuar las respectivas pruebas). Si dentro del trabajo no se realizaron pruebas de hipótesis, dado que el estudio fue a nivel descriptivo o exploratorio, entonces debe de hacerse esta precisión en el proyecto mismo.

\subsection{Diagrama de variables}

En este apartado debe presentarse en forma esquemática, lógica y cronológica, la relación supuesta entre las variables, definiendo la(s) independiente(s) y dependiente(s).

\subsection{Indicadores de las variables}

El concepto de indicador se refiere a aquellos aspectos medibles que nos muestran cómo es el comportamiento de las variables. En el caso de las variables que representan procesos solo se deben incluir y definir los indicadores que sean sujetos de medición.

\subsection{Operacionalización de variables}

Se recurre para ello a una matriz de doble entrada, conocida como Matriz de Consistencia. En ella se presentan, a modo de resumen, las ideas fuerza de cada uno de los componentes de la investigación, según el cuadro adjunto.

\begin{tabular}{|c|c|c|c|c|c|}
\hline \multicolumn{6}{|c|}{ MATRIZ DE CONSISTENCIA } \\
\hline TÍTULO:.. & & & & & \\
\hline \multicolumn{2}{|c|}{ PROBLEMA } & \multicolumn{2}{|c|}{ INVESTIGACIÓN } & \multicolumn{2}{|c|}{ OPERACIONALIZACIÓN } \\
\hline Planteamiento & $\begin{array}{l}\text { Formulación y } \\
\text { Sistematización }\end{array}$ & Objetivos & Hipótesis & Variables & Indicadores \\
\hline & & & & & \\
\hline & & & & & \\
\hline & & & & & \\
\hline
\end{tabular}




\section{Capítulo IV: Metodología de la Investigación.}

Este capítulo comprende la descripción de la estrategia a seguir expresada a través del tipo y modalidad de la investigación, la caracterización de las unidades de análisis, las técnicas, procesos e instrumentos de medición a ser utilizados, los cuales se expresarán en los siguientes apartados:

\subsection{Tipo de investigación}

Este apartado debe reflejar la manera de cómo se enfocó la investigación en cuanto al propósito, amplitud y profundidad, mencionando las características propias del nivel o modalidades de investigación que se aplicó en base a los planteamientos de algunos autores de textos actualizados. Cuando el tipo de investigación demande la aplicación de algún diseño, será necesario exponerlo y explicarlo en cada uno de sus elementos y procesos dentro de este apartado.

\subsection{Población y Muestra}

Aquí deben describirse detalladamente las características propias del conglomerado de sujetos u objetos hacia los cuales se orientó la investigación, es decir, a la totalidad de elementos que podrían ser objeto de medición.

Cuando no se trabaje con toda la población sino con una parte de ella (muestra), debe definirse si ésta fue tomada en forma probabilista, aleatoria o por conveniencia. Si responde al primer caso, debe determinarse un tamaño muestral calculado mediante probabilidades y niveles de confianza definidos. Si la selección fuese aleatoria o por conveniencia, debe justificarse el por qué se hizo de esta manera, reconocer las limitaciones que esto implica y los criterios que se definieron para escoger a los sujetos. Para cualquiera de los casos, debe describirse el proceso de selección de los elementos que conformaron la muestra.

\subsection{Materiales y Métodos}

Descripción detallada de cómo fue realizada la investigación y los elementos utilizados en la misma. Incluye el detalle de procedimientos: explicación general sobre aspectos como número de grupos, asignación de sujetos a grupos, manipulación, etc.

\section{Capítulo V: Tratamiento de los Resultados}

5.1 Técnicas aplicadas en la recolección de la información. Instrumentos de medición.

En este apartado deben explicarse cada una de las técnicas que se aplicaron (entrevista, en- cuesta, observación o alguna otra), a quiénes se les aplicó, con qué propósito, cómo se desarrolló, pasos que se siguieron, y cuál fue el uso específico que se le dio a la información o datos recopilados a través de éstos.

También deben describirse los instrumentos que se han utilizado en la investigación, mencionando cómo es su estructura, su contenido, en qué se han basado para su construcción, cómo se califican y ponderan las respuestas, y cuál ha sido el proceso de validación.

\subsection{Resultados}

En este apartado se debe exponer desde cómo se tabularon y organizaron los datos, los cuadros y gráficos resultantes con la respectiva descripción de los resultados, los modelos estadísticos y/o programas que se utilizaron para su exposición, la justificación del por qué se usaron y cómo se efectuaron las pruebas de hipótesis en el caso que se realicen.

\subsection{Discusión de resultados}

Autoevaluación de los procedimientos empleados y discusión de los posibles alcances y significados de la investigación. Se hace una interpretación acerca de los resultados obtenidos con respecto a la información encontrada en la revisión de la literatura, evitando hasta dónde sea posible especular.

\section{CONCLUSIONES}

Las conclusiones, en el informe de investigación, hacen referencia a los resultados concretos que se obtuvieron en el desarrollo de la investigación y que fueron presentados ampliamente en el desarrollo del cuerpo del trabajo; prácticamente es un resumen sintético de los puntos más importantes y significativos para el autor. Éstos deben ser acordes con el número de objetivos planteados en la investigación, lo cual tampoco implica que no se presenten otras informaciones importantes obtenidas durante el estudio.

\section{RECOMENDACIONES}

Las recomendaciones constituyen el apartado del documento donde la creatividad del investigador se pone de manifiesto en el planteamiento de políticas, estrategias, modelos, instrumentos y medidas de acción a tomar por las organizaciones o instituciones (públicas o privadas) materia de 
estudio, como parte de la solución del problema que se investigó.

\section{BIBLIOGRAFÍA}

Es la última parte del informe de investigación. Agrupa todas las fuentes consultadas para la realización de la investigación y redacción del informe. Las fuentes pueden ser libros, revistas, boletines, periódicos, páginas de Internet, documentos escritos o electrónicos, etc.

Cuando la bibliografía utilizada en el trabajo comprende otras fuentes, además de libros, se dividirán en secciones que se clasifican y ordenan de la siguiente manera: libros, revistas, boletines, periódicos, documentos y otras fuentes (como por ejemplo la entrevista, referencias electrónicas o de Internet). Los titulados de estas secciones irán en mayúscula y subrayado.

Lo que se presenta a continuación es una sugerencia para elaborar la bibliografía, sin embargo a los lectores se les sugiere consultar las Normas que la American Psychological Association (APA) $5^{\circ}$ edición ha elaborado para lograr una bibliografía más completa. Cada fuente se enumera y se ubica en orden alfabético.

Elementos que componen la bibliografía:

\section{LIBROS}

\subsection{Primera opción.}

Los autores deben referenciarse, según este orden, guardando el orden alfabético:

- Apellido paterno del autor en mayúscula y primer nombre separado por una coma, seguido de un punto.

- Título de la obra en cursiva, seguido de un punto. No se deben utilizar las comillas.

- Sólo a partir de la segunda edición se precisará el número de edición, seguido de un punto.

- Ciudad de edición, seguido de un punto.

- Editorial, seguido de un punto. Eliminar las referencias de S.A., Ltda., Cía, etc.

- Año de publicación, seguido de un punto.

- Número de páginas.

Ejemplo:

- BERNAL, Cesar. Metodología de la Investigación para Administración y Economía. Santa Fe de Bogotá. Pearson Educación. 2000. 278 pp.
- ASHLEY, Ruth. Anatomía y Terminología Dental. 2da. Edición. México DF. Editorial Limusa. 1979. 430p.

Si el libro ha sido escrito por dos autores:

- HILL, Charles y Garth JONES. Administración Estratégica. 3ra. Edición. Santa Fe de Bogotá. McGraw-Hill. 1996. 540p.

Si el libro ha sido escrito por tres o más autores:

- HERNANDEZ, Roberto y Otros. Metodología de la Investigación. 3ra. Edición. Santiago de Chile. McGraw- Hill Interamericana. 2004. 706p.

Si el autor es una Institución:

- AMERICAN MANAGEMENT ASSOCIATION. Un nuevo concepto de la Dirección Administrativa. 2da. Edición. Ohio, EEUU. South-Western Publishing. 1976. VI. 80p.

\subsection{Segunda opción}

Debe incluir la referencia completa de todas las obras citadas en el texto, ordenadas alfabéticamente, según el presente modelo.

- Apellido.

- Nombre.

- Fecha.

- Título.

- Editorial.

- Ciudad.

Ejemplo:

- BERNAL, César.

2000 Metodología de la Investigación para Administración y Economía. Pearson Educación. Santa Fe de Bogotá.

Si la bibliografía incluye más de una entrada del mismo autor, éstas deben ir ordenadas según la fecha de publicación.

Ejemplo:

- KOTLER, Philip.

1994a Dirección de la Mercadotecnia. Prentice Hall Hispanoamericana. $7^{\mathrm{ma}}$ edición. México DF.

1994b Fundamentos de Mercadotecnia. Prentice Hall Hispanoamericana. $2^{\text {da }}$ edición. México DF. 


\section{REVISTAS}

- Articulista como autor, seguido de un punto.

- Título del artículo entrecomillado, seguido de un punto.

- Nombre de la revista en cursiva, seguido de un punto.

- Ciudad de edición, seguido de un punto.

- Año y número de la revista, seguido de un punto.

- Editor de la revista.

- Fecha de la revista, seguido de un punto.

- Página primera y última del artículo.

Ejemplo:

- TITO, Pedro. "Gestión del Conocimiento, un nuevo Paradigma Organizacional”. En: Gestión en el Tercer Milenio. Lima. Año 5. № 9. Instituto de Investigaciones de la Facultad de Ciencias Administrativas-UNMSM. Octubre 2002. páginas 119 al 125 .

- ERNST \& Young. "Gestión por competencias, Innovación en la gestión empresarial". En: Cuadernos Cinco Días. Madrid. Año XII. Fascículo $\mathrm{N}^{\circ}$ 6. Páginas 12 al 18.

Referencia de la revista sin autor:

- “Nitroven primera empresa de El Tablazo". En: Revista Momento. Año XVI. № 858. Caracas. Diciembre 1992. Páginas 45 a 48.

\section{TESIS}

- Tesista como autor.

- Título de la tesis en cursivas.

- Indicar tipo de documento (Tesis) y grado para el que se presenta.

- Universidad de procedencia.

Ejemplo:

- BECERRA, Rudy. Nuevos Principios de Gestión en la Industria que trabaja a pedido, con procesos unitarios y variación en los estándares. Tesis (Doctorado). Universidad Nacional Mayor de San Marcos.

\section{BOLETINES}

- Nombre del boletín

- Título del artículo.

- La palabra Boletín.
- Lugar, país.

- Institución responsable.

- Año.

- Número.

- Fecha, página.

Ejemplo:

- Boletín de Ciencias Económicas. Análisis crítico de las diversas interpretaciones del subdesarrollo, punto de vista latinoamericano. Boletín. San Salvador. El Salvador, Universidad José Simeón Cañas. Año X. № 1 enero - febrero de 1987. Páginas 37 a 48.

\section{PERIÓDICOS}

- QUIÑONES, Nilton. "Retroceso en el Presupuesto 2007". En: La República. Martes 28 de noviembre del 2006. p.16.

Si la referencia es del propio periódico, sin especificar el autor:

- LA REPUBLICA. "Necesaria la Independencia Intelectual para alcanzar la Independencia Económica”. En: La República. Año XXV. № 9096. Lima. Domingo 26 de noviembre del 2006. Opinión. p. 36.

\section{INTERNET}

- http://www.adobe.com (Adobe Systems Incorporated)

- http://www.nuevaempresa.cu/03_21.thm.

Si se conoce específicamente la procedencia:

- BUENO, Edgar. "El Capital Intangible como Clave Estratégica en la Competencia Actual". 1998. EN: http://www.gestiondelconocimiento. com/index.php3

- PUZUETA DE PAREDES, Fernando: "Los primeros pasos hacia la Gestión del Conocimiento". 2000. EN: http://www.gestiondelconocimiento. com/casos_area_profesional

\section{ANEXOS}

Aquí se ubicarán los instrumentos y otros tipos de documentos que fueron necesarios para el desarrollo del trabajo, que no se han considerado en otro lugar del informe. Los anexos son secciones adicionales que se adjuntan al documento escrito y deberán ir de acuerdo al orden de importancia que considere el tesista. El objetivo 
es presentar información adicional importante, ya sea para prolongar la explicación de los datos como también para confirmarlos. Se ubica después de las conclusiones, recomendaciones y la bibliografía pertinente.

Ejemplo: copias de documentos, mapas, planos, cuestionarios, guías de entrevista y observación, proyectos, programas, cuadros, gráficos, diagramas, resultados de laboratorios, cronogramas, presentación de la exposición, modelos de aplicación, etc. Dichos agregados son ordenados de acuerdo a cómo han sido citados en el cuerpo del trabajo.

\section{LAS NOTAS O CITAS DE PIE DE PÁGINA}

$\mathrm{Si}$ se desea que el marco teórico goce de credibilidad académica debe contener una abstracción profunda y una bibliografía actualizada, guardando en su redacción consistencia y coherencia lógica.

Para las citas bibliográficas que se utilicen, debe precisarse lo siguiente:

- Las citas de pie de páginas más comunes son: resumen, comentarios textuales y de ampliación.

- En el caso de notas o citas al pie de la página de ampliación, éstas permiten sustentar, definir, especificar significados, referir autores y sus respectivas obras e informar.

- Las citas de pie de página permiten determinar, en cierta forma, la capacidad del investigador, su pensamiento con el de otras personas, y los niveles de abstracción alcanzados.

- Las notas y citas de pie de página amplían la información sin interrumpir la lógica del discurso.

- Las locuciones latinas más utilizadas son:

a) Ibid., ídem, ibídem, equivalen a lo mismo.

b) Op. cit. u Opere Citato equivale a obra citada.

c) Loc. cit. indica lugar citado

\subsection{Modo de utilización}

Las citas se escriben en cursiva, evitando utilizar negrita, ni tampoco subrayado. La numeración de las citas debe ser continua y escrita a espacio simple o sencillo. Se presentan dos opciones de cómo utilizar citas bibliográficas.

\section{a. Primera opción}

Las notas se ubican al pie de página, escritas en Times New Roman, tamaño 10. Sólo se escribirá en cursiva el título del libro, revista, etc. Es opcional poner editorial, ciudad y el año porque en la bibliografía debe aparecer dicha información.

Cuando se cita por primera vez a un autor, la información se coloca de la siguiente manera:

- Autor (apellido y nombre).

- Título de la obra (cursiva).

- Página adonde aparece la cita.

Ejemplo de un solo autor:

1 ROJAS, Raúl. Investigación social. p. 16

2 BOUSQUIEU, Georges. Cómo se deben redactar los informes. p. 14-16.

3 TAMAYO, Augusto. "Lírica Quechua". En: San Marcos. Lima. Vol 1, № 1, Julio-Agosto 2001. p.125. (notas de artículos de revista)

Ejemplo de dos o más autores:

1 GONZALES, Antonio y Domingo MAZA. Tratado Moderno de la Economía General. p.72

Si se trabaja en MS Word, hay una opción fácil y directa de acceder a las notas o citas de pie de página. Para ello sigue el procedimiento siguiente: en la barra de menú abra la ventana insertar. En seguida, al ubicarse en referencia, se abre otra ventana. Dar click en nota al pie, automáticamente el cursor se ubica en el pie de página, listo para escribir los datos que se desea insertar.

b. Segunda opción

Las citas se escribirán en cursiva, sin utilizar negrita ni subrayado. No se emplearán comillas. La numeración de las citas debe ser continua y escrita a espacio simple o sencillo. Cite la fuente en una referencia parentética.

Ejemplo:

$Y$ este mes de octubre, mes del Señor, el trabajo se triplica en cuenta corriente de los fabricantes de la tela morada, en la cuenta de dueños de fábricas de velas, de cerveza, de pasteleros que hacen turrón de Doña Pepa, de joyeros que hacen milagros de plata y de los empresarios de corridas de toros. "En 
Octubre, gracias al Señor, hacen su agosto". (Reynoso 1994:34)

Si la cita de un texto no excede de las cinco líneas, se señala con comillas y se incorpora al texto. No debe escribirse en cursiva.

\subsection{Referencia para notas sucesivas de una misma obra o artículo}

- Ibid. Se utiliza cuando se cita a un mismo autor y a una misma obra, variando solamente la página. Se usa para evitar repetir datos de la fuente.

Ejemplo:

${ }^{1}$ ROJAS, Raúl. Investigación social. p. 16

${ }^{2}$ Ibid. p. 47

- Idem. Se utiliza para identificar el mismo autor, obra y página.

Ejemplo:

1 FERNÁNDEZ, José. El Proceso Administrativo. p.84

2 Idem.

- Loc. cit. / Locus Citato. Indica el texto o lugar citado. Se emplea cuando se repite la información del mismo autor, el mismo libro y las mismas páginas.

Ejemplo:

1 FERNÁNDEZ, José. El Proceso Administrativo. p. 84.

2 ANDER-EGG, Ezequiel. Repensando la investigación acción participativa. p. 70.

3 FERNANDEZ, José. Loc. cit.

- Op. Cit. / Opere Citato. Se emplea cuando se cita más de una vez el mismo autor y obra.

Ejemplo:

1 ANDER-EGG, Ezequiel. Repensando la investigación acción participativa. p. 70

2 SORIANO, Raúl. Investigación social. p.16

3 ANDER-EGG, Ezequiel. Op. cit p. 21.

Como Stoner explica "la organización es un complejo sistema en el que combinando el capi- tal, con los recursos materiales y la inteligencia del colaborador, genera valor para los grupos de interés" (2001: 456)

Los ejemplos antes mencionados constituyen aspectos generales de esta técnica. El objetivo de lo presentado es que los principiantes en la investigación desarrollen los primeros pasos en este amplio y profundo campo.

\section{BIBLIOGRAFÍA}

Huamán, Miguel A. (2002). "Cómo escribir un artículo científico". En: Boletín del Consejo Superior de Investigaciones. Lima, Universidad Nacional Mayor de San Marcos. № 44. Enero- Marzo 2002. Páginas 15 al 22.

Hernández, Roberto y Otros. (2004). Metodología de la Investigación. Tercera Edición. Santiago de Chile, McGraw- Hill Interamericana, $706 \mathrm{p}$.

Artiles, Leticia. (1995). "El artículo científico". En: Revista Cubana de Medicina General Integral. Habana, abril-junio.

Badal, Mauricio. (1990). Elaboración de referencias y citas según las normas de la American Psychological Association (APA), Quinta Edición. Washington.

Cassany, Daniel. (1996). Reparar la escritura. Didáctica de la corrección de lo escrito. Cuarta Edición. Barcelona, Edit. Graó.

Caballero, Alejandro. (1990). Metodología de la Investigación Científica. Segunda Edición. Lima, Edit. Técnico Científica, 180 p.

Day, Ray. (1990). "Cómo escribir y publicar trabajos científicos". En: Revista Organización Panamericana de la Salud (OPS). Washington, páginas 8 al 34 .

Polit, Dense y Hungler, Bernadette. (1997). Investigación científica en ciencias de la salud. Quinta Edición. México DF, Mc-Graw Hill Interamericana.

Walker, Melissa. (2000). Cómo escribir trabajos de Investigación. Barcelona, Edit. Gedisa. 